\title{
High-speed AFM for 1x node metrology and inspection: Does it damage the features?
}

\author{
Hamed Sadeghian*a,b, Teun C. van den Doola, Yoram Uzielc ${ }^{\mathrm{c}}$, Ron Bar Or

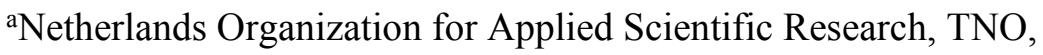 \\ Stieltjesweg 1, 2628 CK Delft, Netherlands; \\ bepartment of Precision and Microsystems Engineering, Delft University of Technology, \\ Delft, The Netherlands \\ ${ }^{c}$ Applied Materials, 9 Oppenheimer Street, Rehovot, 76705, Israel
}

\begin{abstract}
This paper aims at unraveling the mystery of damage in high speed AFMs for 1X node and below. With the device dimensions moving towards the $1 \mathrm{X}$ node and below, the semiconductor industry is rapidly approaching the point where existing metrology, inspection and review tools face huge challenges in terms of resolution, the ability to resolve 3D, and throughput.

In this paper, we critically asses the important issue of damage in high speed AFM for metrology and inspection of semiconductor wafers. The issues of damage in four major scanning modes (contact mode, tapping mode, non-contact mode, and peak force tapping mode) are described to show which modes are suitable for which applications and which conditions are damaging. The effects of all important scanning parameters on resulting damage are taken into account for materials such as silicon, photoresists and low K materials.

Finally, we recommend appropriate scanning parameters and conditions for several use cases (FinFET, patterned photoresist, HAR structures) that avoid exceeding a critical contact stress such that sample damage is minimized.

In conclusion, we show using our theoretical analysis that selecting parameters that exceed the target contact stress, indeed leads to significant damage. This method provides AFM users for metrology with a better understanding of contact stresses and enables selection of AFM cantilevers and experimental parameters that prevent sample damage.
\end{abstract}

Keywords: AFM, atomic force microscope, cantilever, tip sample interaction, wafer, resist, damage, metrology, inspection.

\section{INTRODUCTION}

Metrology and inspection of $1 x$ node wafers and masks currently still is a challenge, especially when high throughput is required. Optical and electron microscopy are currently the work horses but have their limitations.

Scanning Probe Microscopy (SPM) and specifically Atomic Force Microscopy (AFM), due to the advantages of subnanometer resolution and the ability of true 3D scanning, are considered as alternative technologies for metrology, inspection and review of $1 \mathrm{X}$ node and below. Recent developments at TNO have led to a substantially increase of the speed of the measurement, hence, the process throughput [1-5]. A potential disadvantage of AFM can be that substrate or tip damage can occur because of tip-sample interactions. Since AFM is a contact or semi-contact method, depending on the chosen scanning mode, strong interaction forces between the tip and the features on a wafer exist. Due to nanometer size of the tip the indentation area is very small and results in very high Hertzian stresses. The contact stress depends on numerous parameters including scanning parameters such as amplitude of vibration, speed of the measurement and gains of the feedback controller, but also sample and AFM cantilever properties. Therefore, if appropriate parameters are not selected the contact stress exceeds one of the failure stresses and therefore damage can occur. Damage can present itself in the form of fracture, indentation, plastic deformation, buckling and collapsing of the features, tip wear, etc.

* hamed.sadeghianmarnani@tno.nl; phone+3108886 64355; web: tno.nl

Metrology, Inspection, and Process Control for Microlithography XXIX, edited by Jason P. Cain,

Martha I. Sanchez, Proc. of SPIE Vol. 9424, 94240Q · (c) 2015 SPIE

CCC code: $0277-786 \mathrm{X} / 15 / \$ 18 \cdot$ doi: $10.1117 / 12.2085668$

Proc. of SPIE Vol. $942494240 Q-1$ 
We have analyzed the forces that interact between cantilever tip and scanned (wafer) surface and come to the conclusion that in a wide range of operational parameter settings, AFM can be used without causing damage to wafers.

\section{SELECTION OF AFM MODE}

One of the main applications of AFM is to measure the topography (height map) of a surface. There are several modes in which AFM can be operated. They can be explained using a graph of a typical force-distance interaction curve, see Figure 1. Attractive VanderWaals forces are dominant at distances in the order of $10 \mathrm{~nm}$. At distances smaller than $1 \mathrm{~nm}$ repulsive Hertzian contact forces become dominant. It is this repulsive force that can be high and can cause damage.

Several parameters have to be selected for any AFM mode: cantilever dimensions, which determine mass and stiffness, which in term determine the cantilever eigen frequency $\mathrm{f}_{0}$. The damping or quality $(\mathrm{Q})$-factor, which together with the eigen frequency determines the measurement bandwidth $\sim \pi \times \mathrm{f}_{0} / \mathrm{Q}$. The amplitude of vibration, both initially and the amplitude ratio set-point (as a percentage of the initial free-air amplitude) when in contact with the surface. Finally the tip radius has to be selected. A small tip radius gives good resolution but high contact forces. The speed of AFM has been improved to a few $\mathrm{mm} / \mathrm{s}$ [3], and it is envisioned that future systems will be capable of scanning at speeds as high as $100 \mathrm{~mm} / \mathrm{s}$. We will be considering an AFM system which is laterally scanning with this future speed of $100 \mathrm{~mm} / \mathrm{s}$ on a surface with features i.e. steps or lines but also scratches or contaminating particles. These features can have dimensions that exceed $100 \mathrm{~nm}$ height. These scanning conditions result in only one mode being suitable for scanning without damage. This conclusion is solely based on the characteristics of the modes (see Figure 1):

1. Contact / static mode: In this mode the low-stiffness $(\sim 0.1 \mathrm{~N} / \mathrm{m})$ cantilever is snapped-in to the surface and the cantilever is not vibrating while dragging over the surface. The deflection of the cantilever is kept constant with feed-back control. The signal going to the actuator is a measure of the surface topology. Because the cantilever is snapped-in very close to the surface $(<1 \mathrm{~nm})$, it will damage any features on the surface during scanning/dragging. So this mode is not suitable for metrology of vulnerable structures.

2. Tapping / AM (Amplitude Modulation) mode: In tapping mode the cantilever is vibrated at its eigen frequency (typically $1 \mathrm{MHz}$ ) with an amplitude in the order of $100 \mathrm{~nm}$. If the cantilever approaches the surface, the amplitude decreases. A feed-back controller maintains the amplitude ratio at a certain percentage of the freeair amplitude (e.g. 80\%). Because the cantilever is tapping, snap-in is prevented, and the contact time and tapping forces can be kept low. Tapping mode is also suitable for scanning in ambient conditions when almost always a liquid layer will form on the surface. Tapping will penetrate this layer and still detect the real surface and features. Because the (vertical) tapping amplitude is relatively large, it can 'jump' on and over structures on the surface. So this mode can be suitable for metrology and inspection of vulnerable features.

3. Non-contact / FM (Frequency Modulation) mode: In non-contact mode the cantilever is vibrating with a small amplitude (typically $<1 \mathrm{~nm}$ ). A feed-back controller keeps the cantilever at a distance where the VanderWaals forces are dominant. It is often used in non-ambient (vacuum or submersed in water) because liquid nano-layers can shield a clear image of the surface. Because this mode operates at short distance from the surface it cannot jump on or over features so it will cause damage during scanning / dragging. So this mode is not suitable for metrology of vulnerable features.

4. Peak force (tapping) mode: This mode is similar to tapping mode however, the cantilever is 'vibrated' far below its eigen frequency, in the order of $1 \mathrm{kHz}$, and in a sinusoidal motion, to prevent excitation of cantilever eigen frequencies. This way the force curve as depicted in Figure 1 can be sampled. The consequence of this slow tapping is that the cantilever drags along the surface for quite a long time, in the order of $1 \mathrm{us}(100 \mathrm{~nm})$ as compared to $10 \mathrm{~ns}(1 \mathrm{~nm})$ for tapping mode. A drag length of $100 \mathrm{~nm}$ at high speed AFM will damage features on the surface. So this mode is not suitable for high-speed metrology of vulnerable features.

The conclusion is that for scanning at lateral speeds of $100 \mathrm{~mm} / \mathrm{s}$ at a surface with features, the only AFM modes that will not cause damage are modes similar to tapping mode. It allows for a high lateral scanning speed, at good vertical resolution, snap-in to the surface doesn't occur, and lateral frictional forces are almost eliminated. In what follows we will analyze the forces and damage involved in tapping mode. 


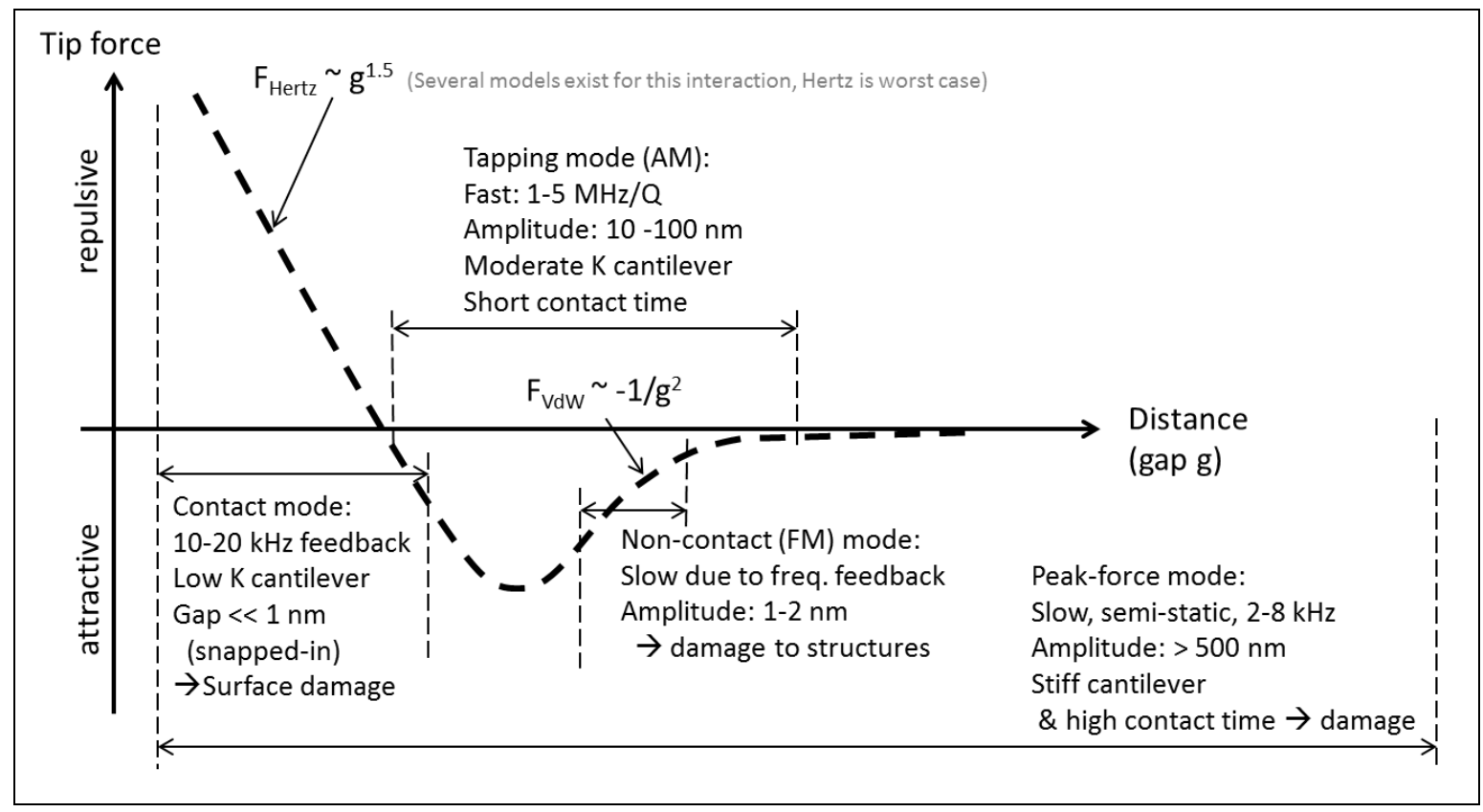

Figure 1: Indication of the distances and settings at which several well-known AFM operation modes are used. For this study Amplitude-Modulation (AM) or tapping mode is analyzed as the only mode which might not cause damage while fast scanning on features.

Table 1. Trade-off between four AFM modes. Damage is not allowed so tapping mode is selected as the only alternative for scanning at $100 \mathrm{~mm} / \mathrm{s}$ over surfaces which can have structures on it.

\begin{tabular}{|l|c|c|c|c|}
\hline Behavior in Mode: & Contact & Tapping & non-contact & Peak force \\
\hline Vertical tapping amplitude & $\sim 0$ & $\sim 100 \mathrm{~nm}$ & $\sim 1 \mathrm{~nm}$ & $>500 \mathrm{~nm}$ \\
\hline Vertical (peak) force & very high & can be low & very low & low \\
\hline Lateral (drag) force & very high & very low & very low & low \\
\hline Contact time (per tap) & continuous & $\sim 20 \mathrm{~ns}$ & none & $\sim 50 \mathrm{us}$ \\
\hline Drag length (per tap at 100 $\mathbf{~ m m / s ~ s c a n n i n g ) ~}$ & continuous & $2 \mathrm{~nm}$ & none & $1 \mathrm{um}$ \\
\hline Damage to structures & damage & can be none & damage & damage \\
\hline Sampling frequency / Speed & very fast & fast (MHz) & slow & slow (<8kHz) \\
\hline Vertical resolution & excellent & very good & excellent & very good \\
\hline Lateral resolution & good & good & very good & good \\
\hline Reach bottom of narrow trenches & good & difficult & good & good \\
\hline Measure physical properties (e.g. elasticity) & no & qualitative & qualitative & quantitative \\
\hline Automation & easy & complicated & complicated & easy \\
\hline
\end{tabular}

\section{ANALYZYNG TIP-SAMPLE INTERACTIONS AND CANTILEVER DYNAMICS}

The dynamic movement of the cantilever is given by the second order differential equation given in formula (1) below in which the left hand term is the standard continuum equation of motion with damping and the right hand term describes the driving forces. The cantilever is driven at its eigen frequency by a piezo with a continuous harmonic excitation $\mathrm{F}_{\mathrm{o}} \sin (\omega \mathrm{t})$. At long distance the main acting (attractive) force is the VanderWaals force, which has a square dependence on distance. At short distance several repulsive forces act of which the Hertzian contact force dominates the peak stress. Capillary forces can be neglected for relative humidity lower than $40 \%$ or if the surface is hydrophobic. The model 
according to formula (1) has been solved numerically using nonlinear difference method to find the peak force for many different parameter settings. It is noteworthy that the electrostatic force according to the Derjaguin-Landau-VerweyOverbeek (DVLO) model has been neglected in this study. The results will be discussed in the next section. It was verified that our calculations gave the same results as reported in literature as a function of, amongst others, amplitude set-point, surface elasticity, and cantilever stiffness [6-8] Error! Reference source not found.Error! Reference source not found.

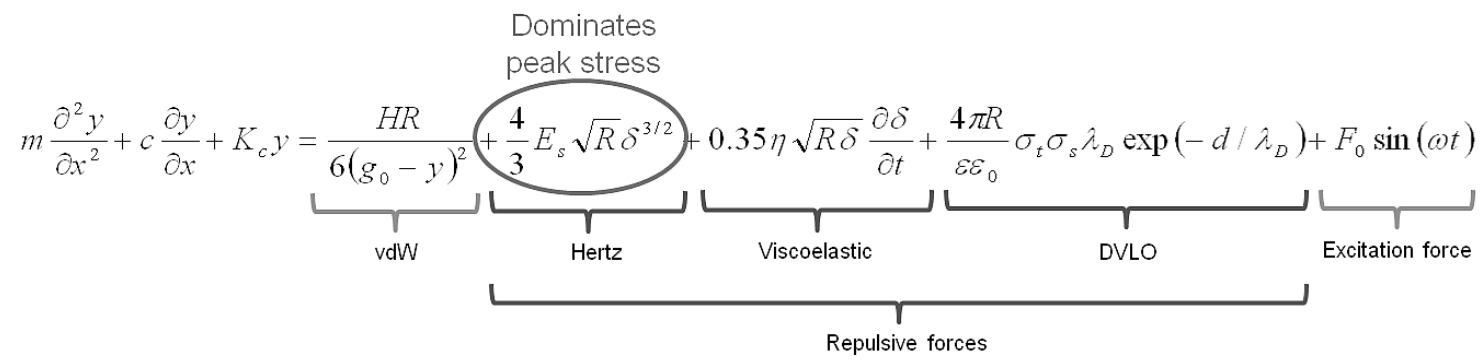

$\mathrm{y}=$ deflection of the cantilever

$\mathrm{g}_{0}=$ Initial gap between surface and cantilever tip, $\mathrm{g}_{0}-\mathrm{y}=$ real gap during tapping

$\mathrm{m}=$ cantilever effective vibrating mass

$\mathrm{c}=$ cantilever damping, which is related to its Q-factor by $Q=\sqrt{m \cdot K_{c}} / c$

$\mathrm{K}_{\mathrm{c}}=$ cantilever effective stiffness

$\mathrm{HR}=$ Hamaker constant

$\mathrm{g}_{0}=$ initial gap between tip and surface

$\mathrm{E}_{\mathrm{s}}=$ effective elasticity between tip and wafer

$\mathrm{R}=$ cantilever tip radius

$\delta=$ sample indentation

$\eta=$ relaxation coefficient of the viscoelastic material

The model was also used to assess the forces if scanning over step-like features. In this case the amplitude set-point changes abruptly from one tap to the next. If the taps are fully elastic then the first step gives the highest stress. During later taps (in the order of several times Q taps) the stress will gradually decrease because the feed-back controller will start retracting the cantilever until the amplitude ratio is equal to the set-point. At a cantilever tapping frequency of $1 \mathrm{MHz}$ and a lateral velocity of $100 \mathrm{~mm} / \mathrm{s}$, the lateral distance between taps is $100 \mathrm{~nm}$. The tip radius typically also is $100 \mathrm{~nm}$. So it is likely that a tap is made on the edge of the step instead on a full flat surface. Equation (1) is solved for full flat surfaces so we analyzed with a Finite Element Method (FEM) how much higher the stress will be if tapping on an edge instead of on a full surface. It appears that the stress can be a factor 3 higher on an edge as compared to tapping on a full surface (see Figure 2).

Note that even if a single tap pushes the stress beyond the yield stress, it normally doesn't cause damage because the duration of each tap is extremely short. Only somewhere between $10^{8}$ and $10^{9}$ taps damage will occur [6]. Especially on soft materials like biological material, the stress can be very high without ever causing damage [7].

Another remark should be made on the calculated Herzian contact stresses (vertical force divided by cross section of indentation). In practice the Von Misses stress is a better approximation of occurring stresses. VonMises takes into account how material deforms in all directions, not only the vertical direction. The Von Misses stress is a factor 1.6 lower than the Herzian stress. So relative to the results reported below, there is an extra safety factor in the order of 1.6 . 


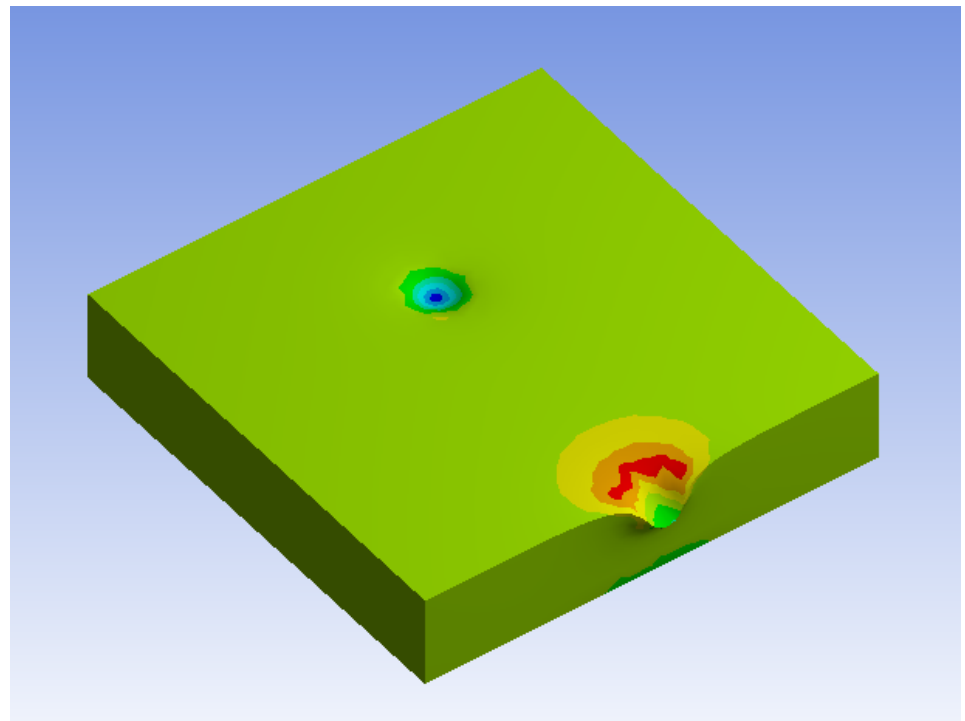

Figure 2: Peak tapping force on bare silicon wafer both for on the full surface and on an edge. The stress on the full surface is $3 \mathrm{MPa}$ and on the edge it is $9 \mathrm{MPa}$. Cantilever tip radius is $100 \mathrm{~nm}$, the $A_{\text {init }}=100 \mathrm{~nm}, \mathrm{~A}_{\text {ratio }}=60 \%, \mathrm{f}=1 \mathrm{MHz}$, and $\mathrm{K}=10 \mathrm{~N} / \mathrm{m}$

Also not taken into account in the analysis is the effect of viscoelastic energy dissipation in the surface material. Especially on soft materials like resist this has a significant effect. The peak force can reduce by $30 \%$. Also the higher vibrational modes of the cantilever will absorb energy ( $20 \%)$ and therefor reduce the peak stress.

We have also used the Tatara contact model [7] instead of the Hertzian contact model, which is more suitable for viscoelastic materials. Figure 3 shows the difference between Hertzian contact and Tatara contact models for a case of photoresists such as SU8. It shows that in reality there is an extra safety factor in the order of 1.4.

All in all the results shown in the following section can be considered worst case. In practice the safety factor or number of taps before damage occurs will be higher. The extra safety factor is in the order of 4 . But on the edge of a feature the stress can be 3 times higher. So then the calculated stresses are approximately valid.

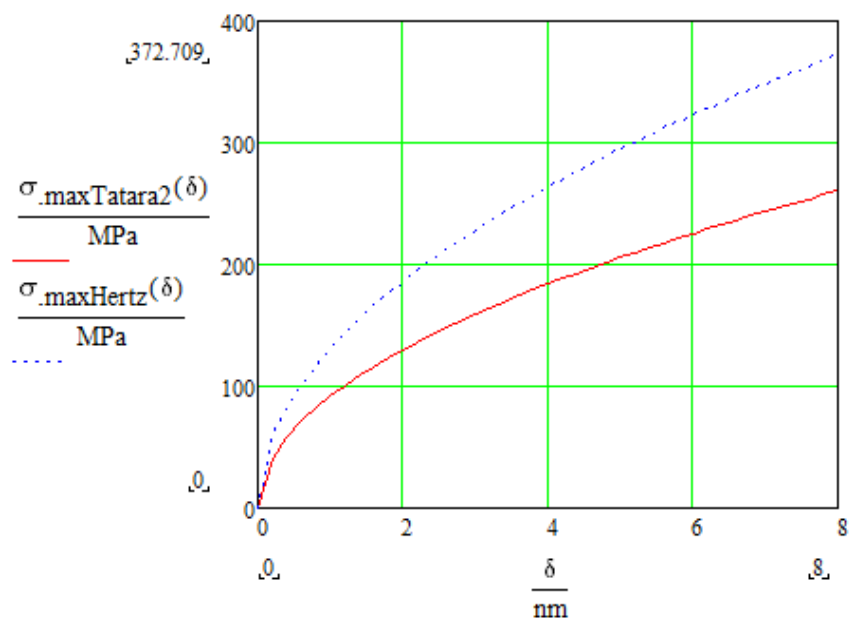

Figure 3: Tatara vs. Hertz contact stress for a photoresist. 


\section{RESULTS OF CASE STUDIES}

The peak tapping force has been numerically solved from formula (1) for the following situations:

1. Bare Silicon surface

2. Surface coated with SU8 resist

3. Step-like features on a Silicon surface

\subsection{Bare Silicon surface}

Figure 4 shows the final optimized result with the effect of some parameter variations. The cantilever stiffness was either 1 or $10 \mathrm{~N} / \mathrm{m}$ with an eigen frequency of $1 \mathrm{MHz}$ and the tip has a radius of $100 \mathrm{~nm}$. A cantilever damping of Q=100 was found to give the lowest peak stresses on Silicon. The Yield stress of Silicon is taken to be $9 \mathrm{GPa}$. The initial free-air cantilever amplitude $\mathrm{A}_{\text {init }}$ was varied between $5 \mathrm{~nm}$ and $100 \mathrm{~nm}$. The peak stress is slightly lower for high amplitude

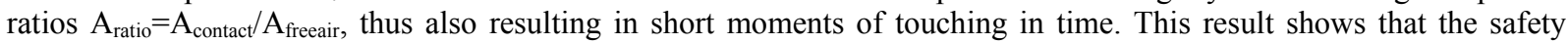
factor before damage on bare silicon wafers is always larger than 10 .

The safety factor gradually increases for smaller cantilever amplitudes (see Figure 5). But at small amplitudes the risk increases of causing damage by dragging features from the surface. So a larger amplitude is preferred. The stress is also lower for lower cantilever stiffness. But for very low cantilever stiffness there is risk of snap-in to the surface. So a cantilever stiffness between 0.1 and $1 \mathrm{~N} / \mathrm{m}$ can be selected as the optimum. A lower damping (higher Q-factor) will give less peak stress (see Figure 5). But lower damping also results in a slower measuring system. So a moderate Q-factor $=100$ is preferred. A large tip radius results in lower stress (see Figure 6). But at very large tip radii the VanderWaals forces increase and the (vertical) sensitivity decreases. A tip radius in the order of $100 \mathrm{~nm}$ is preferred.

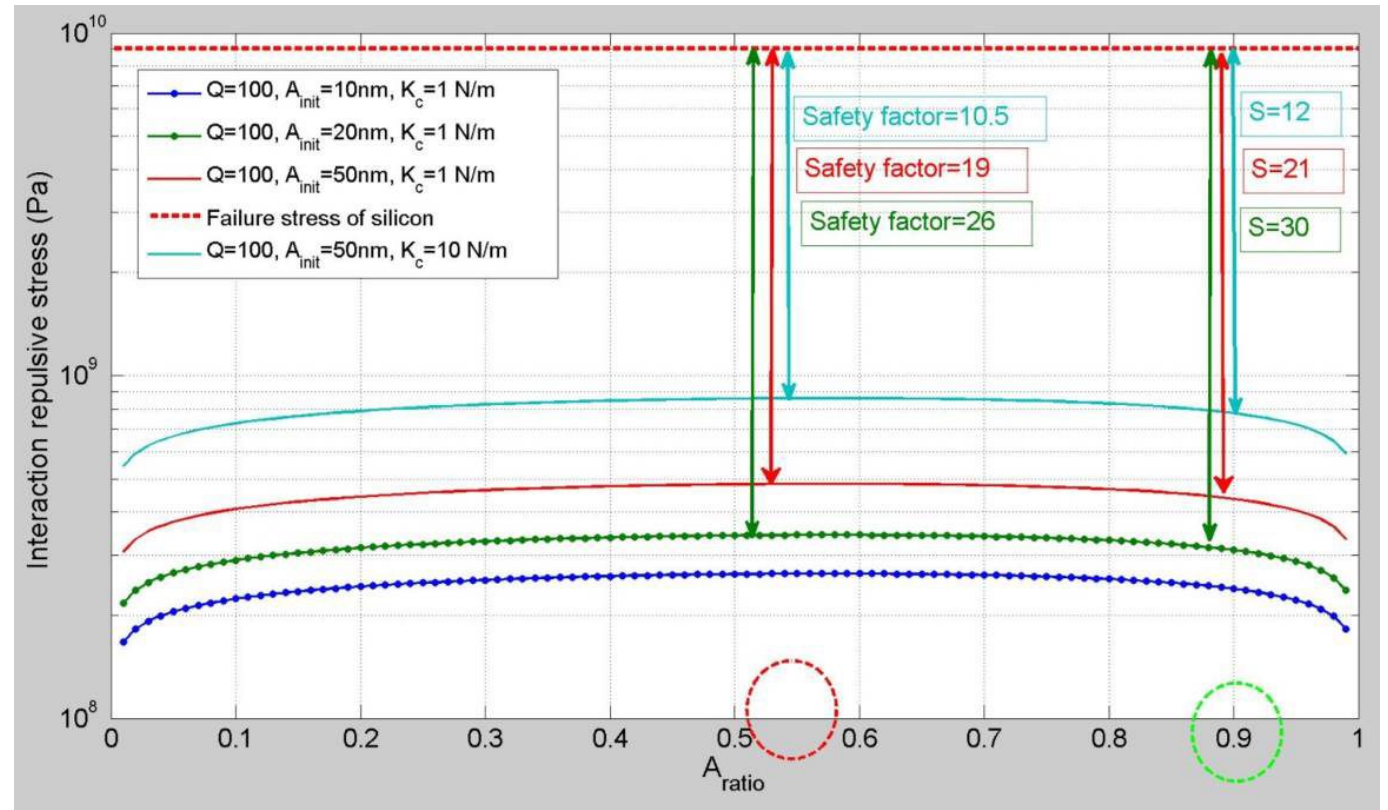

Figure 4: Peak tapping force on bare silicon wafer for several tapping mode parameter settings. 

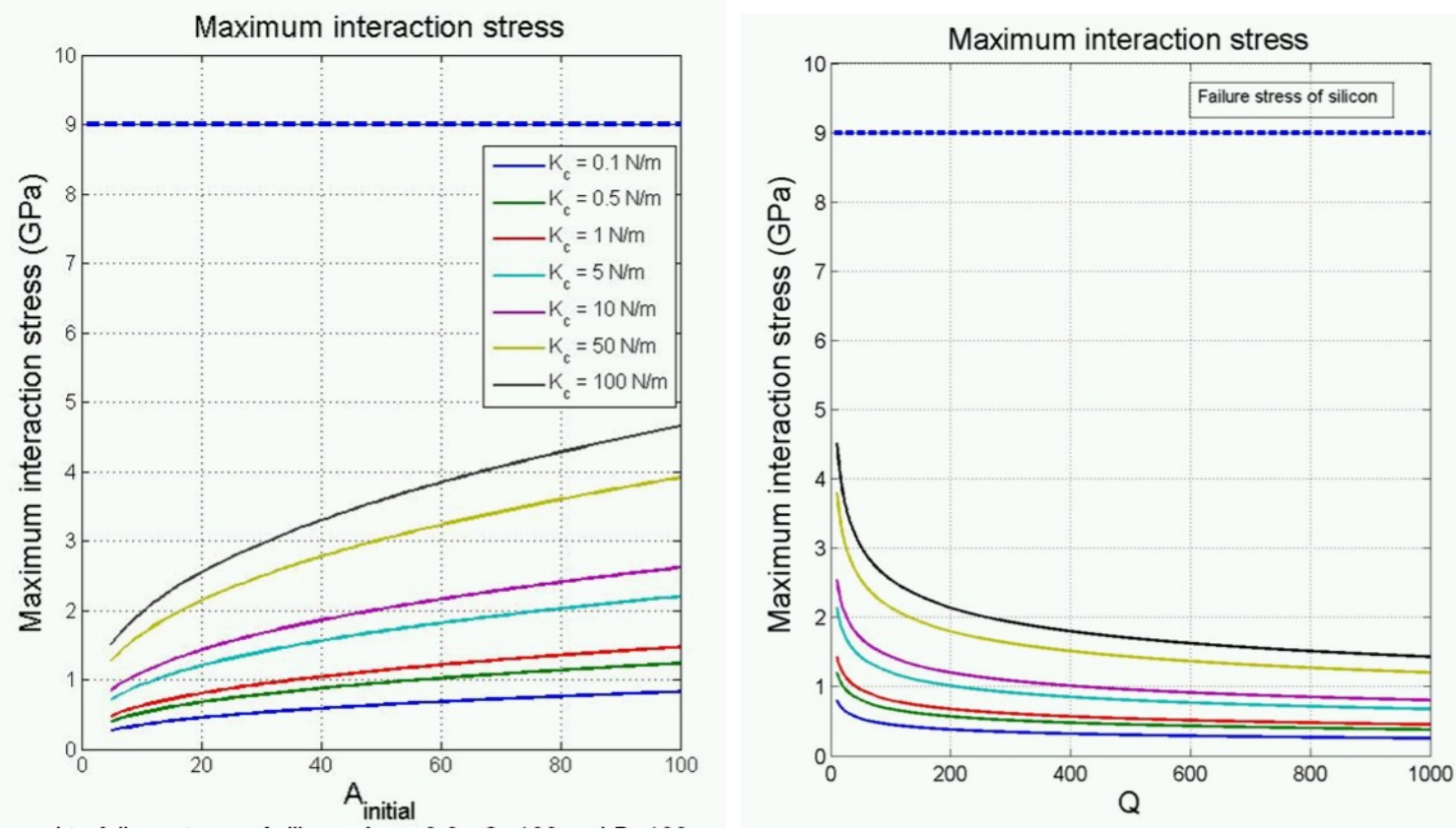

Figure 5: Peak tapping force on bare silicon wafer as a function of cantilever stiffness $\mathrm{K}_{\mathrm{c}}$, damping $\mathrm{Q}$, and initial free-air vibration amplitude $\mathrm{A}_{\text {init. }}$

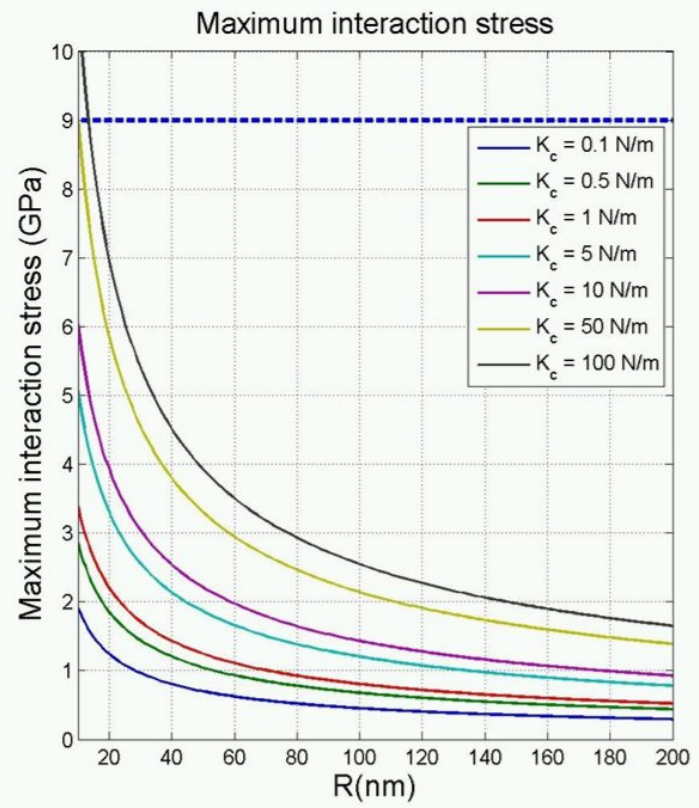

Figure 6: Peak tapping force on bare silicon wafer as a function of cantilever tip radius $\mathrm{R}$ and stiffness $\mathrm{K}_{\mathrm{c}}$. 


\subsection{Surface coated with SU8 resist}

The analysis was repeated for a wafer with resist (SU8). We find that similar parameter settings as for bare wafers give an optimum (low) stress on resist, see Figure 7. In an absolute sense the stresses are lower because resist is more compliant than Silicon. But the yield stress of resist $(80 \mathrm{MPa})$ is a lot lower than for Silicon $(9000 \mathrm{MPa})$. So the safety factor decreases considerably relative to Silicon. But still there is a safety margin in the order of 2 . Thus also scanning on resist won't cause damage.

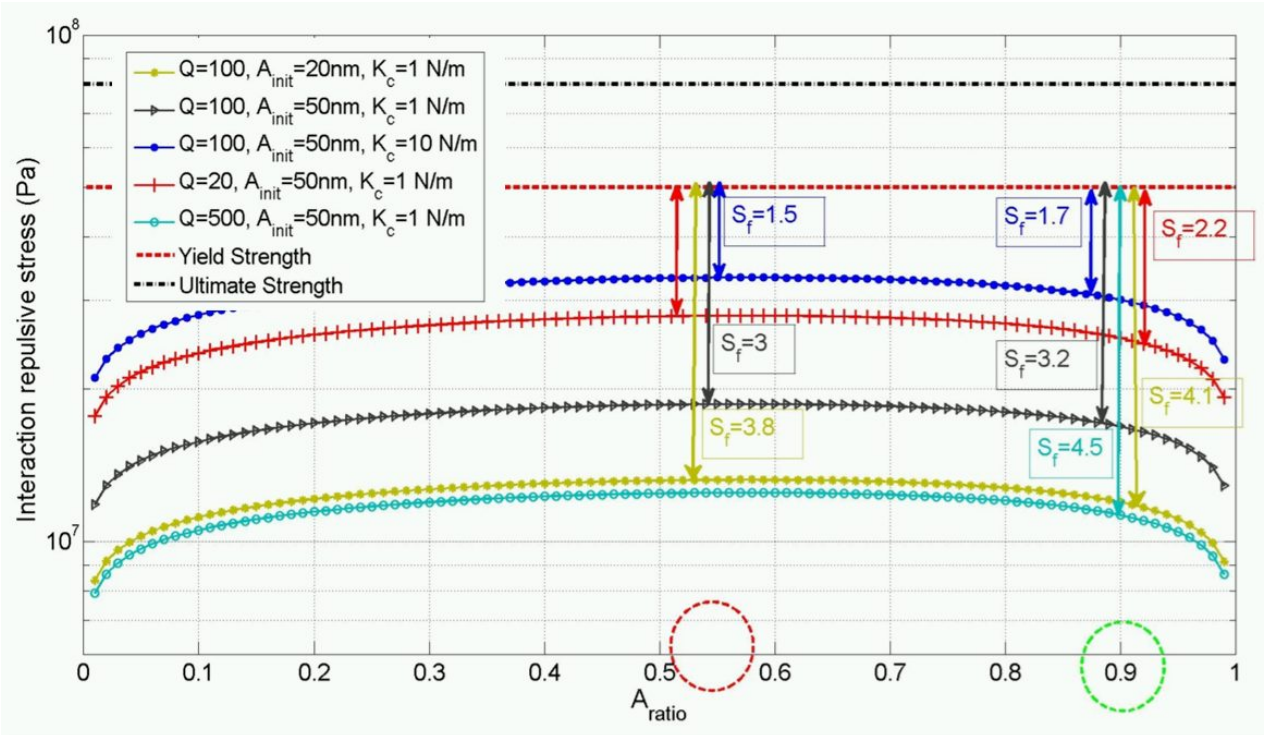

Figure 7: Peak tapping force on a wafer coated with SU8 resist, again for several tapping mode parameter settings.

\subsection{Step-like features on a Silicon surface}

Next we analyzed the peak force if there is a sudden step-like feature while scanning over the surface. Note that as explained in the previous section, this analysis is for the situation where the whole cantilever tip is tapping on the step. If the tap is on the edge of the step, the stress can be up to a factor three higher, but due to several other relaxing factor (see previous section), we are still safe. Figure 8 shows that:

- As in the previous analysis, the lowest stresses occur at an amplitude ratio set-point in the order of $80 \%$.

- A lower cantilever stiffness $\mathrm{K}_{\mathrm{c}}$ gives lower stress. But the cantilever stiffness should be high enough to prevent sticking due to snap-in. Also very soft $(<0.1 \mathrm{~N} / \mathrm{m})$ cantilevers aren't commercially available. So the preferred cantilever stiffness is between 0.1 and $1 \mathrm{~N} / \mathrm{m}$.

- $\quad$ A larger tip radius $\mathrm{R}$ gives lower stress. But a too large radius gives risk of snap-in. $\mathrm{R}=100 \mathrm{~nm}$ is optimum.

- Lower amplitude $\mathrm{A}_{\text {init }}$ gives lower stress but the amplitude should be larger than the step size and sufficiently large to prevent sticking. An amplitude between 50 and $100 \mathrm{~nm}$ is preferred.

For features which are additionally very slender, such as the case for a Fin-FET as depicted in Figure 9, this stress analysis is also valid. We additionally verified that buckling (due to tapping on the top) and bending stress (due to the cantilever pushing laterally against the Fin-FET) were far below the maximum yield stress of Silicon. 


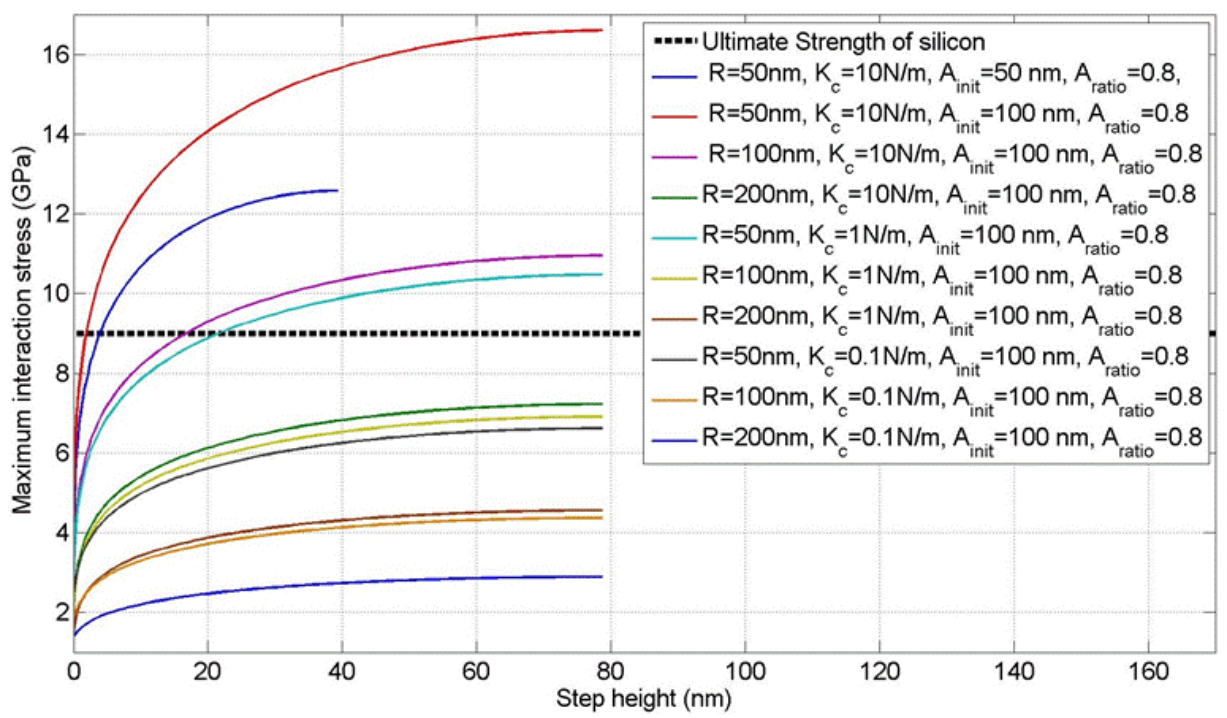

Figure 8: Peak tapping force on a bare Silicon wafer for several parameter settings.
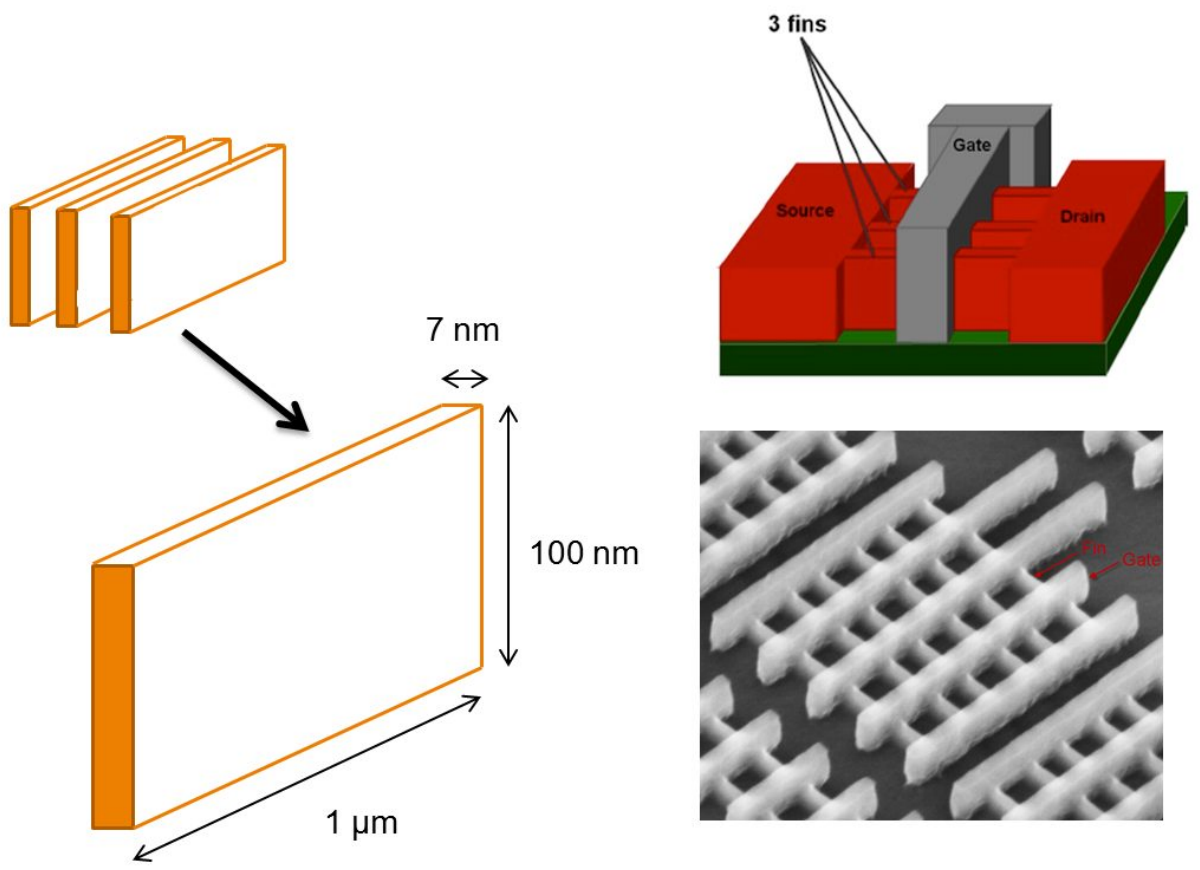

$100 \mathrm{~nm}$

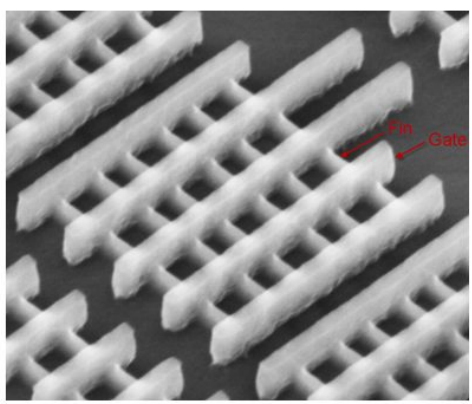

Figure 9: Typical Fin-FET structure. The SEM of the Fin-FET is taken from Intel website. 


\section{CONCLUSIONS}

The worst case scenario is tapping on a soft surface (e.g. resist coated wafer) on top of a feature with an amplitude equal to the height of the feature. Even in this situation the features won't be damaged if proper scanning parameters are selected.

For both bare Silicon wafers, resist coated wafers, and step-like features we found the following parameters to give good scanning detail without resulting in damage:

- Relative high resonant eigen frequency of $1 \mathrm{MHz}$, allowing fast scanning (instead of standard in the order of $100 \mathrm{kHz}$ )

- $\quad$ Average damping of $\mathrm{Q}=100 . .500$

- Relative low cantilever stiffness $K_{c}=0.1$ to $1 \mathrm{~N} / \mathrm{m}$ (instead of standard values of $1-10 \mathrm{~N} / \mathrm{m}$ ) this is a compromise between low stress and risk of snap-in.

- $\quad$ Blunt tip radius of $100 \mathrm{~nm}$ (but not larger to prevent snap-in)

- Large initial free-air vibration amplitude of 50 to $100 \mathrm{~nm}$. This is a compromise between low stress and ability to scan over features.

- Amplitude ratio of $80 \%$ (cantilever vibration amplitude after engaging to the surface $=0.8 \mathrm{x}$ the amplitude in free air).

\section{REFERENCES}

[1] H. Sadeghian, R.W. Herfst, T.C. van den Dool, W.E. Crowcombe, J Winters, "High-throughput Parallel SPM for Metrology, Defect, and Mask Inspection”, Proc. SPIE 9231, 30th European Mask and Lithography Conference, 92310B (2014).

[2] H. Sadeghian, N. B. Koster, T. C. van den Dool "Introduction of a high throughput SPM for defect inspection and process control", Proc. SPIE 8681, Metrology, Inspection, and Process Control for Microlithography XXVII, 868127 (2013).

[3] H. Sadeghian, T. C. van den Dool, W. E. Crowcombe, R. W. Herfst, J. Winters, "Parallel, miniaturized scanning probe microscope for defect inspection and review", Proc. SPIE 9050, Metrology, Inspection, and Process Control for Microlithography XXVIII, 90501B (2014).

[4] R. J. F. Bijster, J. de Vreugd, H. Sadeghian, "Phase lag deduced information in photo-thermal actuation for nanomechanical systems characterization”, Applied Physics Letters, 105(7), 073109 (2014).

[5] R.W. Herfst, W.A. Klop, M. Eschen, T.C. van den Dool, N.B. Koster, H. Sadeghian, "Systematic characterization of optical beam deflection measurement system for micro and nanomechanical systems", Journal of Measurement 56, 104-116 (2014).

[6] Vahid Vahdat and Robert W. Carpick, "Practical Method to Limit Tip-Sample Contact Stress and Prevent Wear in Amplitude Modulation Atomic Force Microscopy" ACS Nano, 2013, 7 (11), pp 9836-9850.

[7] Horacio V. Guzman, Alma P. Perrino, and Ricardo Garcia, Peak Forces in High-Resolution Imaging of Soft Matter in Liquid", ACS Nano, 2013, 7 (4), pp 3198-3204.

[8] Horacio V Guzman, Ricardo Garcia, "Peak forces and lateral resolution in amplitude modulation force microscopy in liquid", J Nanotechnol. 2013 Dec 6;4:852-9. 